\title{
APPLYING MACHINE LEARNT EXPLICIT ALGEBRAIC STRESS AND SCALAR FLUX MODELS TO A FUNDAMENTAL TRAILING EDGE SLOT
}

\author{
R.D. Sandberg; R. Tan, J. Weatheritt, \\ A. Ooi, A. Haghiri \\ Department of Mechanical Engineering \\ University of Melbourne \\ Parkville, VIC 3010, Australia \\ Email: richard.sandberg@unimelb.edu.au
}

\author{
V. Michelassi \\ Baker Hughes, a GE Company \\ Florence 50127, Italy \\ vittorio.michelassi@bhge.com
}

\author{
G. Laskowski \\ General Electric Aviation \\ Lynn, MA 01905, USA \\ laskowsk@ge.com
}

\section{ABSTRACT}

Machine learning was applied to LES data to develop nonlinear turbulence stress and heat flux closures with increased prediction accuracy for trailing-edge cooling slot cases. The LES data were generated for a thick and a thin trailing-edge slot and shown to agree well with experimental data, thus providing suitable training data for model development. A Gene Expression Programming (GEP) based algorithm was used to symbolically regress novel nonlinear Explicit Algebraic Stress Models (EASM) and heat-flux closures based on either the gradient diffusion or the generalized gradient diffusion approaches. Steady RANS calculations were then conducted with the new EASM models. The best overall agreement with LES data was found when selecting the near wall region, where high levels of anisotropy exist, as training region, and using the mean squared error of the anisotropy tensor as cost function. For the thin lip geometry, the adiabatic wall effectiveness was predicted in good agreement with the LES and experimental data when combining the GEP-trained model with the standard eddydiffusivity model. Crucially, the same model combination also produced significant improvement in the predictive accuracy of adiabatic wall effectiveness for different blowing ratios, despite not having seen those in the training process. For the thick lip case, the match with reference values deteriorated due to the presence of large-scale, relative to slot height, vortex shedding. A GEP-trained scalar flux model, in conjunction with a trained RANS model, was found to significantly improve the prediction of the adiabatic wall effectiveness.

\section{INTRODUCTION}

The trailing edge of a high pressure turbine blade, which represents its thinnest geometrical section is exposed to substantial heat load during operation and therefore requires efficient cooling strategies. Trailing edge cooling of high pressure turbine airfoils is typically accomplished with either pressure side bleed slots or trailing edge ejection depending on application, design requirements and cost. A canonical representation of the trailing-edge slot is a wall jet with finite slot lip thickness. This configuration was studied experimentally by Kacker and Whitelaw [1] using a range of blowing and

*Address all correspondence to this author. temperature ratios, slot heights and slot lip thicknesses, and their experimental data have been used for comparison in a number of subsequent numerical studies. Using unsteady RANS (URANS), Holloway, Medic and Durbin [2], Joo and Durbin [3] did find significant differences to the experimental data, in particular in terms of the prediction of the adiabatic wall effectiveness. This was attributed mainly to the inability of RANS modeling to reproduce the correct level of turbulent mixing in the region very close to the wall.

In recent years, turbulence models have been developed with the aid of data-driven methodologies. Broadly speaking, varied machine-learning algorithms are being applied as regression tools to large, high-fidelity data sets to infer closure terms in turbulence closures. For example, neural networks have been applied to secondary flow prediction [4] and Gaussian processes have modeled corrective terms for channel flow [5]. A third and quite different method is gene expression programming [6] (GEP), a form of evolutionary algorithm, which was successfully used to implement new stress-strain relationships for separated flow [7]. The algorithm is a form of symbolic regression, such that the result of the optimization is a tangible equation which can be easily implemented into a flow solver.

Machine learning tools have also been applied to turbomachinery related problems. Recently, a comparison of neural networks and GEP to regress non-linear stress-strain relationships showed very similar predictive performance, yet the GEP optimization was at a fraction of the cost [8]. Further, GEP was applied to high-pressure turbines and showed improvement over the linear model [9]. These studies looked primarily at the stress-strain relationship, yet researchers are also exploring other model components. One group has successfully extracted functional forms for intermittency transport, in order to model bypass transition [10,11]. For heat-transfer problems, similar to that addressed in this paper, random forest regression has been applied to model the turbulent thermal diffusivity, for a gradient-diffusion model in the scalar transport of temperature [12].

In this paper, GEP is used to formulate improved turbulence and heat flux closures, using subsets of highly resolved LES data, for which improved prediction of adiabatic wall effectiveness is achieved. The paper is structured as follows. First the LES and RANS set-ups used throughout this work are detailed. Then the machine-learning approaches for the construction of Reynolds 
stress and heat flux models are presented. Next, the LES results are compared against experimental data [1], along with baseline RANS predictions. Thereafter, the underlying cause for the over-prediction of the adiabatic wall effectiveness of baseline RANS is discussed through an analysis of the LES datasets for two of the cases also studied by Kacker and Whitelaw [1]. The results obtained using the newly trained non-linear models are presented and the sensitivity to the subset of LES data considered is discussed.

\section{COMPUTATIONAL DETAILS}

A sketch of the simulation domain is presented in Fig. 1a. The flow configuration fundamentally represents a two dimensional wall-jet in a co-flow. The inlet velocity profiles were obtained from the experiment results provided by Kacker and Whitelaw [1]. For the present simulations, two different values of non dimensional lip thickness were considered. They represent flows with different physical phenomenon. An overview of the simulation flow parameters are shown in Tab. 1.

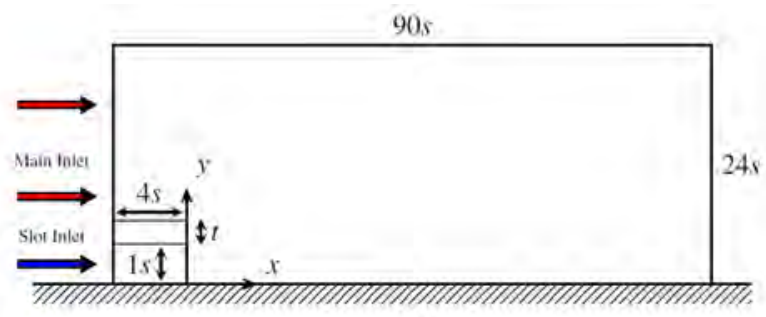

(a)

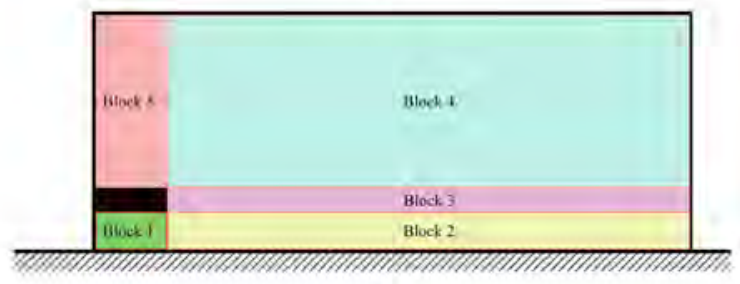

(b)

FIGURE 1: (a) Computational domain (not to scale) and (b) LES blocks.

\section{LES Set-up}

The large eddy simulations were conducted using the in-house multi-block structured compressible Navier-Stokes solver HiPSTAR [13]. The wall adapting local eddy viscosity (WALE) model [14] was used for modeling of the sub-grid scale stresses. In solving the density weighted filtered energy equation, the sub-grid scale heat flux contribution is assumed to be zero. This assumption is valid for sufficiently refined grid such that the sub grid scale heat flux contribution is negligible as compared to the resolved heat flux.

A fourth-order accurate central differencing scheme was used for spatial discretization in the streamwise and wall normal direction. A spectral method based on the FFTW3 library was used for the discretization in the spanwise direction. Additionally, skew-symmetric splitting was used to stabilize the convective terms. As for the temporal discretization, a five-step, fourth-order
TABLE 1: Simulation Parameters

\begin{tabular}{l|l}
\hline Simulation Parameters & Values \\
\hline \hline$U_{\text {bulk }}$ & $24.2 \mathrm{~m} / \mathrm{s}$ \\
\hline$U_{f s}$ & $19.2 \mathrm{~m} / \mathrm{s}$ \\
\hline$I_{\text {slot }}$ & $5 \%$ \\
\hline$I_{\text {main }}$ & $0.5 \%$ \\
\hline$l_{t}$ & $10 \%$ of slot height \\
\hline$B R$ & 1.26 \\
\hline$t / s$ & $1.14 \& 0.126$ \\
\hline$R e_{\text {slot }}$ & $\approx 12,000$ \\
\hline$T_{\text {slot }}$ & $273 \mathrm{~K}$ \\
\hline$T_{f s}$ & $323 \mathrm{~K}$ \\
\hline
\end{tabular}

accurate Runge Kutta scheme was used.

The baseline computational grid of the LES composed of five blocks as illustrated in Fig. 1b. The first block represents the nozzle region and was composed of 80 points in the $x$ and 128 points in the $y$ direction. The second, third and fourth blocks represent the region downstream of the nozzle and all contained 1,280 points in the $x$ direction and in the $y$ direction block 2 had 128 points from the wall to one slot height, block 3 had 128 points or 64 points to represent the slot thickness in the cases $t / s=1.14$ and $t / s=0.126$, respectively, and block 4 had 128 points from the upper slot wall to the freestream boundary at $24 \mathrm{~s}$. The fifth block was composed of 80 points in the $x$ and 128 points in the $y$ direction. For the thicklip and thin-lip cases 32 and 64 Fourier modes were used in the spanwise direction respectively, corresponding to 66 and 130 collocation points. In the LES, only mean profiles were prescribed at the inlet and no fluctuations were added as only marginal differences in the flow and temperature fields had been observed in previous studies [15]. Characteristic boundary conditions were used for the freestream and at the outlet a zonal characteristic boundary condition was employed [16] using 30 streamwise points. All walls were set to no-slip boundaries and adiabatic temperature conditions were used. The non-dimensional timestep was set to $6 \times 10^{-4}$ to satisfy the CFL condition and all cases were initially run for 360 time units, based on slot height and freestream velocity, to overcome the initial transient. The LES were then performed for another 240 time units in order to collect averaged quantities and statistics. Additional LES were conducted on different grids and the LES were run for longer for comparison to ensure that the results were grid independent and statistically converged, respectively.

\section{RANS Set-up}

For the RANS calculations, initially the baseline $k-\omega$ SST turbulence model [17] from OpenFoam version 2.4 was used. The incompressible SimpleFoam and PimpleFoam solvers were used for steady RANS and unsteady RANS calculations, respectively. These solvers couple the incompressible Reynolds averaged momentum equation with the Poisson equation to solve for the velocity and pressure field respectively. The use of the incompressible solvers 
can be justified as follows. Firstly, the free-stream velocity and maximum jet velocity is well below Mach 0.3 . Secondly, small temperature differences between the jet flow and the free-stream imply that buoyancy effect can be neglected from the mean momentum equation. Thus, the energy equation can be decoupled from the momentum equation, allowing for the temperature field to be solved passively. The inlet conditions of the RANS simulations, i.e. the values of turbulence intensities and the turbulent length scale, $l_{t}$, were taken from Ivanova and Laskowski [15]. From these turbulent length scale, the turbulence intensity and specific dissipation prescribed at the inlet were computed with the following relations

$$
k_{\text {inlet }}=\frac{3}{2}(U I)^{2}, \quad \omega_{\text {inlet }}=\frac{k_{\text {inlet }}^{1 / 2}}{c_{\mu}^{1 / 4} l_{t}} .
$$

This resulted in the free-stream and slot inlet turbulent intensity to be set as $0.5 \%$ and $5 \%$, respectively.

For spatial discretization, second order central differencing schemes were used. The spatial Gauss linear scheme in OpenFoam, a second order central differencing scheme was being used for discretization for most of the flow variables except for the temperature and turbulent quantities used in RANS model. The divergence term of the passive scalar transport equation was discretized using the linearUpwind scheme in OpenFoam. The order of accuracy of this scheme correspond to somewhere between a first and second order accurate scheme. As for the turbulent quantities, both the modeled form of the turbulent kinetic energy and the turbulent specific dissipation were being discretized using a Gauss upwind scheme (1st order accurate) for the divergent terms in their respective transport equation. The gradient and Laplacian terms in the respective momentum and Poisson equation were discretized using the Gauss linear schemes. In the case of the Unsteady RANS calculation, the time discretization of the unsteady term in the momentum equation uses the implicit, second order backward Euler schemes.

The computational grid of the RANS was conducted using the same resolution in the $x-y$ plane as in the LES. However, the RANS calculations were conducted in two dimensions only, rather than in 3D as the LES. The baseline computational grid of the thicker lip thickness to slot ratio case has a total grid count of 510,306 cells in the $x-y$ plane while the case with the smaller lip thickness to slot ratio has a total grid count of 428,321 cells in the $x-y$ plane. An OpenFoam tool was used to convert the structured grid used in the LES to a unstructured grid with approximately the same total cell count. A one cell thick grid is used in the span-wise direction for the RANS calculation. The $y^{+}$of both cases were kept well below 0.2 for the first cell away from the wall (lower wall and the lip wall).

\section{Methodology For Reynolds Stress Closure}

The incompressible form of the Reynolds-averaged momentum equation, written as

$$
\frac{\partial \bar{u}_{i}}{\partial t}+\bar{u}_{j} \frac{\partial \bar{u}_{i}}{\partial x_{j}}=-\frac{\partial \bar{p}}{\partial x_{i}}+v \frac{\partial^{2} \bar{u}_{i}}{\partial x_{j} \partial x_{j}}-\frac{\partial \tau_{i j}}{\partial x_{j}}
$$

where overbars denote Reynolds-averaged quantities and the density is absorbed into the modified pressure $\bar{p}$, contains the unclosed term $\tau_{i j}$. Therefore, $\tau_{i j}$, known as the Reynolds stress tensor, must be modeled and as such contributes to a large portion of the error in a RANS calculation. In so called linear RANS models, the Reynolds stress tensor is modeled using the Bousinessq approximation

$$
\tau_{i j}=\frac{2}{3} k \delta_{i j}-2 v_{t} S_{i j}
$$

This dictates that deviations from the isotropic part $\frac{2}{3} k \delta_{i j}$, where $k$ is the turbulent kinetic energy, are linear in the strain rate tensor $S_{i j}$. The constant of proportionality, the eddy viscosity $v_{t}$, is obtained from one or two transport equations [18].

In a common extension to linear modeling, one can append higher order combinations of the strain and rotation rate tensor to the Boussinesq approximation. In the current study using a machine-learning framework, the goal is to model the anisotropy tensor $a_{i j}^{\text {mod }}$ such that

$$
\tau_{i j}=\frac{2}{3} k \delta_{i j}-2 v_{t} S_{i j}+2 k a_{i j}^{m o d}\left(S_{i j}, \Omega_{i j}\right)
$$

where

$$
a_{i j}^{\text {mod }}=\sum_{\lambda=1}^{10} G^{\lambda}\left(I^{1}, I^{2}\right) V_{i j}^{\lambda}\left(S_{i j}, \Omega_{i j}\right) .
$$

$V_{i j}^{\lambda}\left(S_{i j}, \Omega_{i j}\right)$ are integrity basis functions and are polynomial in $S_{i j}$ and $\Omega_{i j}$. Due the the Cayley-Hamilton theorem, there are ten such independent polynomials. The coefficients $G^{\lambda}$ are functions of the scalar invariants $I^{k}$. For two dimensional flows, the number of independent basis functions reduces to three and there are only two independent scalar invariants: $I^{1}=S_{i j} S_{i j}$ and $I^{2}=\Omega_{i j} \Omega_{j i}$. The definitions of the polynomial basis functions are provided in Pope [19].

In order to close Eq. 4, one must provide the coefficients $G^{\lambda}$. Typically, this is achieved through mathematical simplification of the transport equation for the anisotropy of Reynolds stress [18]. However, in this paper we follow a trend in data-driven approaches that aim to fit a functional form to each $G^{\lambda}$ for a given dataset. This can be done through the minimization of the cost function $J$,

$$
J=\frac{1}{N} \sum_{n=1}^{N} \sum_{i=1}^{3} \sum_{j=1}^{i}\left(a_{i j}^{m o d}\left(S_{i j}^{L E S}, \Omega_{i j}^{L E S}\right)-a_{i j}^{L E S}\right)^{2}
$$

$a_{i j}^{L E S}$ is the non-dimensional 'true' anisotropy tensor at each of the $N$ data points in specified 'training regions' of the LES cases described above. Typically training regions correspond to areas the engineer wishes to more accurately capture with their RANS solver. The sensitivity of the training region definitions is discussed in the results section. Equation 6 aims to minimize the mean squared error of the model $a_{i j}^{\text {mod }}$, computed using the LES velocity field. Therefore the process does not require repeated RANS simulations for each new model. Note, in order to preserve dimensionality, the strain and rotation rate tensors must be non-dimensionalized by a turbulent timescale. In this work we choose a definition using the 
specific dissipation rate, such that the timescale is equal to $\frac{1}{\omega}$. In order to obtain $\omega$ we passively solve the $\omega$ transport equation once, by freezing the LES velocity and Reynolds stress fields [20]. The result is a dissipation rate consistent with the LES fields and the RANS transport equations. For more information on this process see earlier work on turbine blade flows [9].

To minimize Eq. 6, Gene Expression Programming [6] (GEP), a type of evolutionary algorithm, is used to symbolically regress the three coefficients $G^{1,2,3}\left(I^{1}, I^{2}\right)$. GEP evolves a population of candidate functions, expressed as trees - which are decoded into mathematical equations (example outputs are given in Eq. 16). GEP employs analogies to survival of the fittest, using Eq. 6 as a measure of individual fitness, and genetic mutation, which modifies the tree structure of candidate models. As the algorithm progresses, incremental changes through mutation occur with the poor changes filtered out by natural selection. The output is the best solution in the population after a specified number of iterations. As the algorithm is symbolic, no functional form is specified by the user. Instead, a functional form is evolved through increasingly complicated tree structures. The algorithm is non-deterministic due to the randomness inherent in mutation and this allows for the training of many models. For more information on evolutionary algorithms see the work of Koza [21] and Ferreira [6]. For the specifics of tailoring the algorithm to turbulence modeling, see Weatheritt and Sandberg's initial implementation [7].

\section{Methodology for Scalar Flux Closure}

In an incompressible framework, temperature can be considered a passive scalar, the mean transport of which is governed by the equation

$$
\frac{\partial \bar{T}}{\partial t}+\bar{u}_{j} \frac{\partial \bar{T}}{\partial x_{j}}=\frac{v}{\operatorname{Pr}} \frac{\partial^{2} \bar{T}}{\partial x_{j} \partial x_{j}}-\frac{\partial \overline{u_{j}^{\prime} T^{\prime}}}{\partial x_{j}}
$$

The heat flux term is usually modeled using the gradient diffusion hypothesis,

$$
\overline{u_{i}^{\prime} T^{\prime}}=-\alpha_{t} \frac{\partial \bar{T}}{\partial x_{j}}
$$

which dictates the scalar flux points in the direction of maximum mean temperature gradient and the model acts as a turbulent diffusion term. The diffusivity $\alpha_{t}=\frac{v_{t}}{P r_{t}}$ is modeled as eddy viscosity over turbulent Prandtl number $\operatorname{Pr}_{t}$. In this study, instead of using a turbulent viscosity by constant turbulent Prandtl number assumption, the machine-learning framework, outlined in the previous section, is used to infer the thermal diffusivity as a function of velocity gradients and temperature $\alpha_{t}=\alpha_{t}\left(T, S_{i j}, \Omega_{i j}\right)$, much like an existing study for jets in crossflow [12].

A second formulation for $\overline{u_{i}^{\prime} T^{\prime}}$ is also considered, which can be considered an extension of the first. Eq. 8 assumes an isotropic turbulent diffusivity. Instead, a generalized gradient diffusion hypothesis (GGDH) [22] can be assumed

$$
\overline{u_{i}^{\prime} T^{\prime}}=-f \frac{1}{\omega} \tau_{i j} \frac{\partial \bar{T}}{\partial x_{j}},
$$

where $f=f\left(T, S_{i j}, \Omega_{i j}\right)$ is a scalar field to be optimized. This form is found by assuming that the scalar flux is proportional to its rate of production with the implicit terms neglected.
This gives us two optimization problems, namely closure via $\alpha_{t}$ and closure via $f$. In both cases, the cost function

$$
J=\frac{\sum_{n=1}^{N}\left(\phi^{m o d}-\phi^{L E S}\right)^{2}}{\sum_{n=1}^{N}\left(\phi^{L E S}\right)^{2}}
$$

is used, where $\phi$ is either $\alpha_{t}$ or $f$. The target quantity $\phi^{L E S}$ for each optimization is found by contracting the respective equations with the temperature gradient (Eqs. $8 \& 9$ ),

$$
\begin{gathered}
\alpha_{t}^{L E S}=-\left(\frac{\overline{u_{i}^{\prime} T^{\prime}} \frac{\partial \bar{T}}{\partial x_{i}}}{\frac{\partial \bar{T}}{\partial x_{j}} \frac{\partial \bar{T}}{\partial x_{j}}}\right)^{L E S}, \\
f^{L E S}=-\left(\frac{\omega \overline{u_{k}^{\prime} T^{\prime}} \frac{\partial \bar{T}}{\partial x_{k}}}{\tau_{i j} \frac{\partial \bar{T}}{\partial x_{i}} \frac{\partial \bar{T}}{\partial x_{j}}}\right)^{L E S} .
\end{gathered}
$$

The models $\phi^{\text {mod }}$ are found by constructing independent invariants from $\frac{\partial \bar{T}}{\partial x_{j}}, S_{i j}$ and $\Omega_{i j}$. The full list used in this study closely mirrors the work of Milani et al. [12] and is given as

$$
\begin{aligned}
& I^{1}=S_{i j} S_{j i}, \quad I^{2}=\Omega_{i j} \Omega_{j i}, \quad I^{3}=S_{i j} S_{j k} S_{k i}, \quad I^{4}=\Omega_{i j} \Omega_{j k} S_{k i}, \\
& I T_{1}=\frac{\partial \bar{T}}{\partial x_{i}} \frac{\partial \bar{T}}{\partial x_{i}}, \quad I T_{2}=\frac{\partial \bar{T}}{\partial x_{i}} S_{i j} \frac{\partial \bar{T}}{\partial x_{j}}, \quad I T_{3}=\frac{\partial \bar{T}}{\partial x_{i}} S_{i j} S_{j k} \frac{\partial \bar{T}}{\partial x_{k}}, \\
& I T_{4}=\frac{\partial \bar{T}}{\partial x_{i}} \Omega_{i j} \Omega_{j k} \frac{\partial \bar{T}}{\partial x_{k}}, \quad I T_{5}=\frac{\partial \bar{T}}{\partial x_{i}} \Omega_{i j} S_{j k} \frac{\partial \bar{T}}{\partial x_{k}}, \quad I T_{6}=\frac{\partial \bar{T}}{\partial x_{i}} \Omega_{i j} S_{j k} S_{k l} \frac{\partial \bar{T}}{\partial x_{l}}, \\
& I T_{7}=\frac{\partial \bar{T}}{\partial x_{i}} \Omega_{i j} S_{j k} \Omega_{k l} S_{l m} \frac{\partial \bar{T}}{\partial x_{m}}, \quad I T_{8}=\frac{\partial \bar{T}}{\partial x_{i}} \Omega_{i j} \Omega_{j k} S_{k l} \frac{\partial \bar{T}}{\partial x_{l}},
\end{aligned}
$$

where the velocity gradient is non-dimensionalized by $1 / \omega$. The temperature gradient is non-dimensionalized by $\frac{k^{0.5}}{c_{\mu} \omega}$, where $\omega$ is obtained through the same process outlined in the previous section. Therefore all quantities are non-dimensional in Eq. 13 and only non-dimensional quantities can be made from them. This implies to ensure dimensional consistency, $\alpha_{t}^{L E S}$ is multiplied by $\frac{\omega}{k}$. The GEP algorithm is tasked with making functions for $\alpha_{t}^{\text {mod }}$ and $f^{\text {mod }}$ out of Eq. 13. For this, the standard GEP algorithm is used [6].

\section{Results and Discussion}

Instantaneous temperature fields of the LES results for both the thick and thin lip cases are presented in Fig. 2. For a view of the mean behavior, in particular the mean spreading of the mixed fluid region, time-averaged temperature contours are also added to the figures with the levels chosen as $T_{\text {slot }}+2 K$ and $T_{f s}-2 K$. The threshold of $\pm 2 K$ was chosen somewhat arbitrarily but was found to display the spreading of the thermal wake very well, with only marginal differences to even smaller threshold values. From the instantaneous colour-contours, it can be seen that the vortices that form behind the thicker lip wall are significantly larger than those generated by the thin lip. These larger-scale structures lead to increased turbulent mixing bringing the hot main-path flow towards the wall much earlier than in the thin-lip case, with the first occurrence of increased wall temperature seen in the range $10 \leq x / s \leq 15$. 


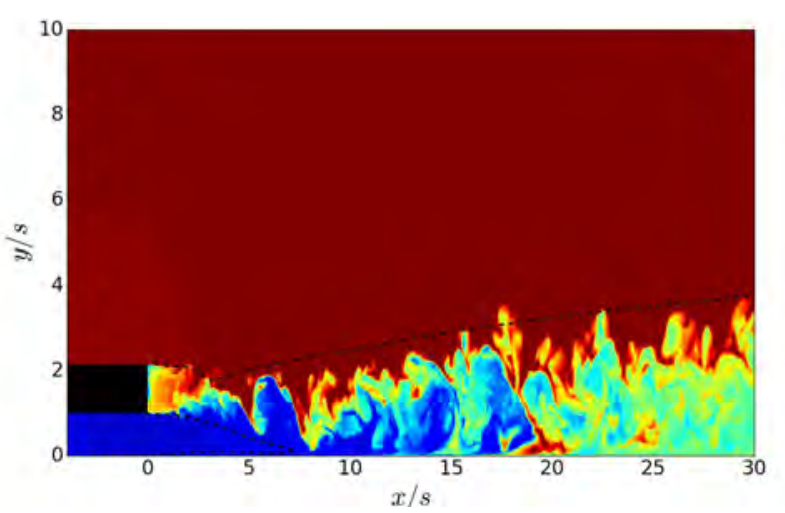

(a) Instantaneous LES temperature field for $t / s=1.14$.

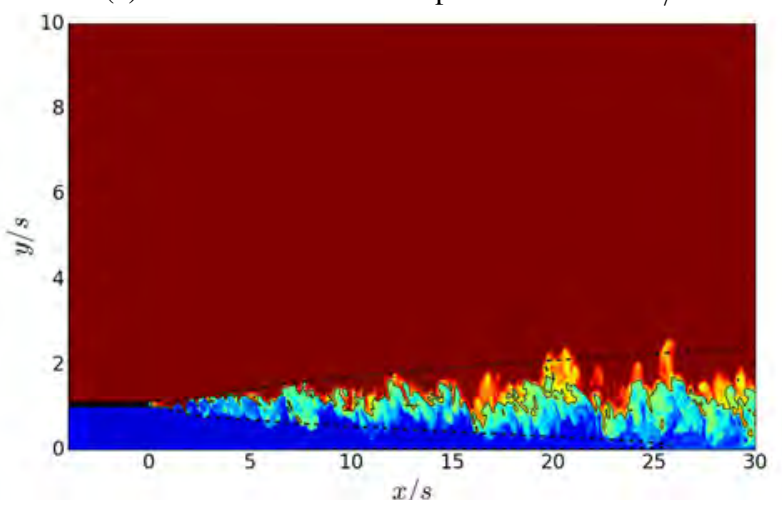

(b) Instantaneous LES temperature field for $t / s=0.126$.

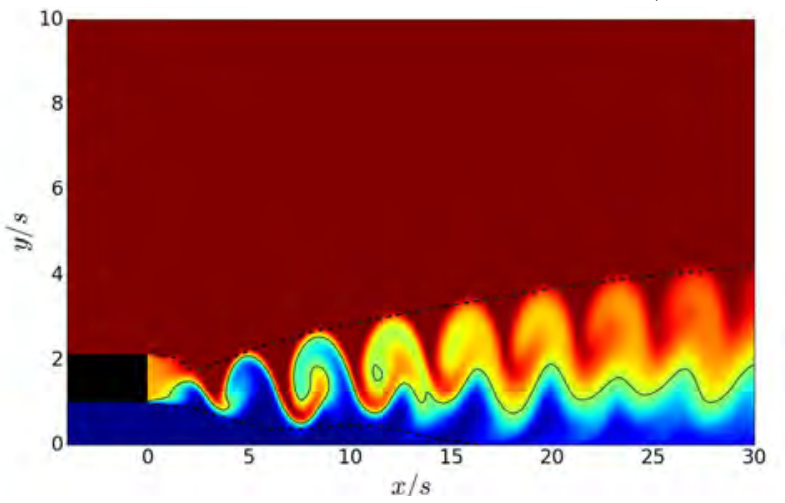

(c) Instantaneous URANS temperature field for $t / s=1.14$.

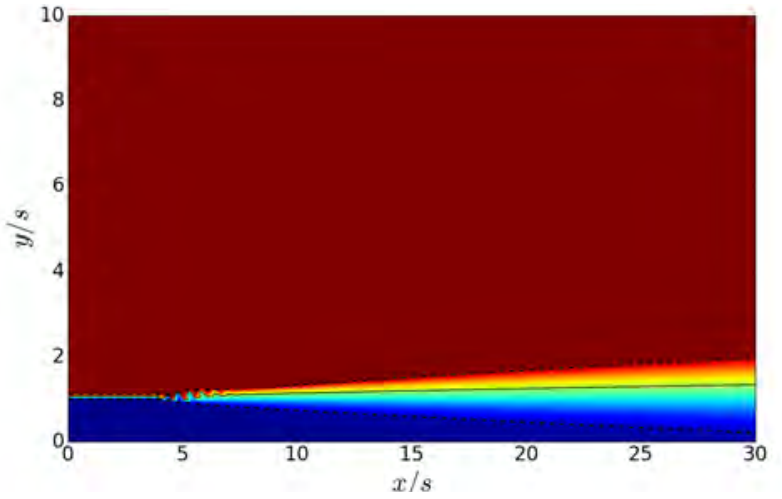

(d) Instantaneous URANS temperature field for $t / s=0.126$.

FIGURE 2: Instantaneous temperature fields obtained from LES and unsteady RANS using the baseline $k-\omega$-SST model, dashed lines denote time-averaged temperature at $T_{s l o t}+2 \mathrm{~K}$ and $T_{f s}-2 \mathrm{~K}$.

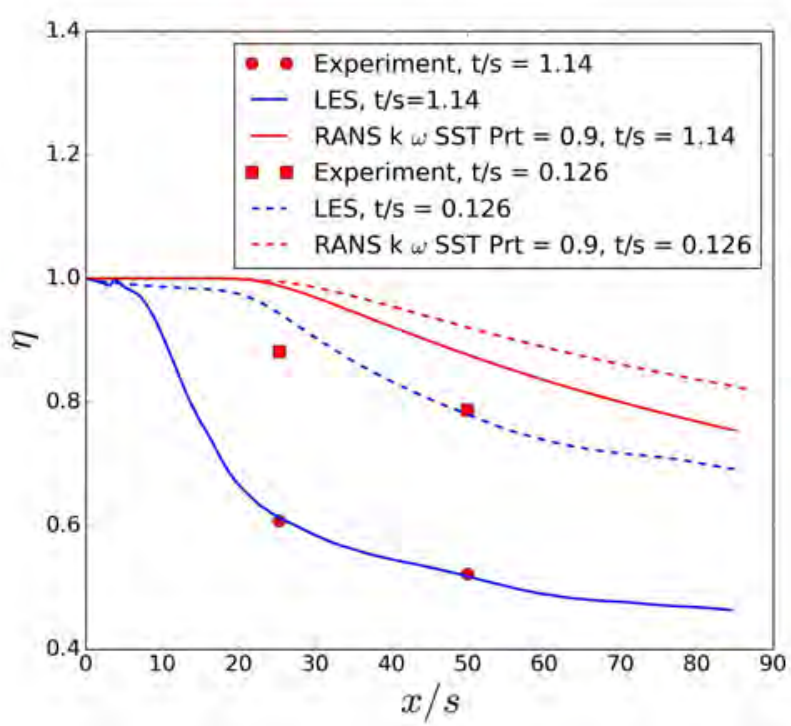

FIGURE 3: Comparison of $\eta_{\text {wall }}$ obtained from LES, experiments [1] and baseline RANS for different $t / s$.

The mean temperature contour at $T_{\text {slot }}+2 \mathrm{~K}$ confirms that the cooling flow is rapidly mixed with the freestream flow and thus the temperature at the wall has increased by $2 K$ already at $x / s \approx 7$. For the case with the thinner lip-geometry, the turbulent mixing does not affect the wall until at least 25 slot heights downstream of the trailing edge, as reflected by the sudden drop in the adiabatic wall effectiveness (see Fig. 3) and the mean temperature contour at $T_{\text {slot }}+2 \mathrm{~K}$ that extends to just beyond $x / s=25$. Results obtained from unsteady RANS using the baseline $k-\omega$ SST turbulence model and the standard eddy-diffusivity model for the heat flux are shown for comparison in Fig. $2 \mathrm{c}$ and $2 \mathrm{~d}$, again including the time averaged temperature contours with levels $T_{\text {slot }}+2 K$ and $T_{f s}-2 K$. For the thick-lip case $(t / s=1.14)$, large scale vortex shedding can be observed persisting over a long streamwise distance and mixing the jet flow with the external flow. Although the mean contour at $T_{f s}-2 K$ is in reasonable agreement with the one in the LES, the mean temperature contour with level $T_{\text {slot }}+2 K$ shows a very different behavior to the LES data, in that cool flow with $T \leq T_{\text {slot }}+2 \mathrm{~K}$ persists much further downstream due to a shallower slope of the contour line in the near nozzle region. In the thin-lip case, vortex shedding with significantly smaller spatial scales with a quick return to a steady flow at around $x / s=8$ is observed. Again, the contour line of mean temperature at $T_{\text {slot }}+2 \mathrm{~K}$ exhibits a shallower slope than in the LES, hence leading to a longer region where a low temperature is maintained at the wall. However, this effect is by far not as pronounced as in the thick-lip case. These results re-emphasize that the two chosen test cases feature significant differences in their physical behavior and part of the current study is to assess whether turbulence and heat transfer closures can be trained for steady calculations that are robust to these very disparate cases.

A comparison of the adiabatic wall effectiveness between the linear steady RANS (k- $\omega$ SST model), LES results and the experimental result of Kacker and Whitelaw [1] are presented in Fig. 3. Since the value of the $\operatorname{Pr}_{t}$ strongly influences the wall temperature due to variation in the heat transfer diffusivity, the value of $\mathrm{Pr}_{t}$ is kept constant at a commonly assumed value of 0.9 in the current 


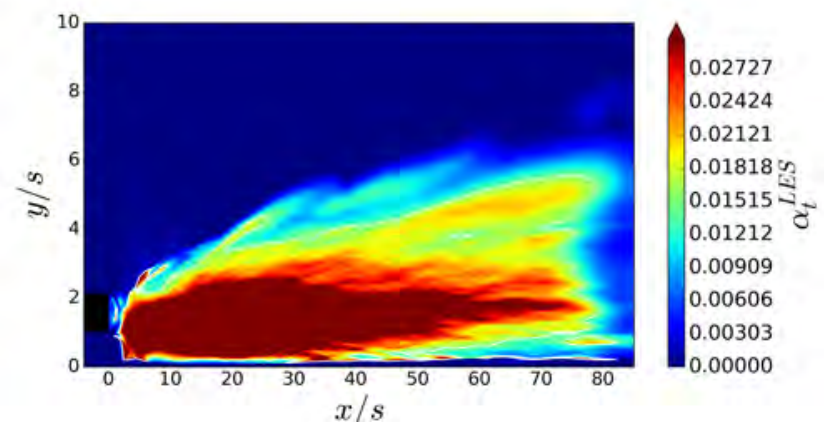

FIGURE 4: Thermal diffusivity obtained from LES using equation (11).

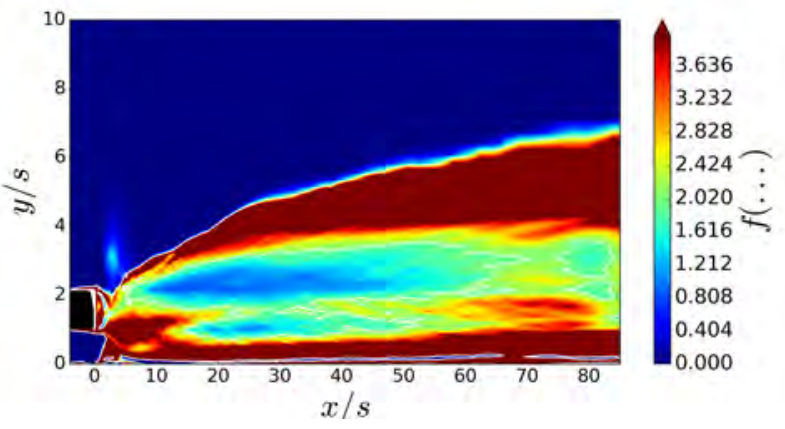

FIGURE 5: Thermal diffusivity obtained using GGDH, equation (12).

study. The results show that the highly resolved LES are generally in good agreement with the reference experimental data. Thus, the LES data can be seen as a reliable data set representing the physics of the simplified trailing-edge slot with sufficient accuracy to be used for RANS model assessment and modelling using the above discussed machine-learning strategy. The RANS calculations using the baseline model on the other hand severely over predict the adiabatic wall effectiveness. There are several reasons contributing to this over prediction. Firstly, one mechanism driving both the momentum and scalar diffusion can be attributed to the eddies generated at the interface of the shear layer between the jet and the freestream/near wall flow. This mechanism is not represent in steady RANS modeling. Another possible source of deficiency is the eddy-diffusivity model used in the Reynolds averaged advection diffusion equation. In this case, the model error is related to both its mathematical formulation as well as the assumption of a constant turbulent Prandtl number. The use of such a model assumes an isotropic turbulent diffusivity at each point in the flow and the connection between the momentum and scalar diffusion via a constant turbulent Prandtl number is questionable in general. Equations (11) and (12) are used to evaluate the thermal diffusivity or $G G D H$ coefficient, respectively, predicted by the LES. As can be seen in Figs. 4 and 5, results obtained from either approach exhibits significant spatial variation which implies that heat flux modeling cannot be neglected in this application.

Thirdly, the over prediction of the adiabatic wall effectiveness can be attributed to the inability of the linear RANS model to reproduce the high level of near wall anisotropy as compared to the LES approach. Using the LES data, this can be quantified by considering the alignment of the strain rate tensor and the anisotropy tensor,

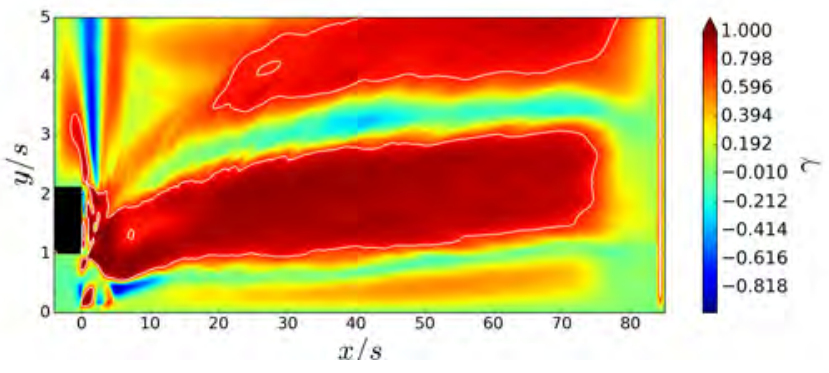

(a) $t / s=1.14$

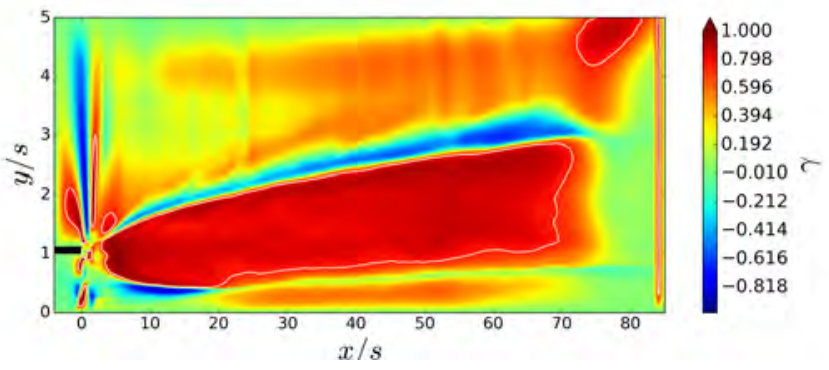

(b) $t / s=0.126$

FIGURE 6: Alignment of strain and anisotropy tensor using baseline $k-\omega$-SST model.

\begin{tabular}{l|l|l|l}
\hline Model Name & Training Region & LES & Eqn. \\
\hline \hline KCI.R.1b & $0<x / s<85,0<y / s<1$ & $t / s=1.14$ & $16 a$ \\
\hline KCI.R.thin.1 & $0<x / s<85,0<y / s<1$ & $t / s=0.126$ & $16 b$ \\
\hline KCI.R.1f & $0<x / s<85,0<y / s<3$ & $t / s=1.14$ & $16 c$ \\
\hline HF.1 (GDH) & $30<x / s<85,0<y / s<3$ & $t / s=1.14$ & $17 a$ \\
\hline HF.2 (GGDH) & $30<x / s<85,0<y / s<3$ & $t / s=1.14$ & $17 b$ \\
\hline HF.3 (GGDH) & $0<x / s<30,0<y / s<3$ & $t / s=1.14$ & $17 c$ \\
\hline
\end{tabular}

TABLE 2: Model Names and Training Region/ Dataset

computed as follows [23]

$$
\begin{gathered}
a_{i j}^{L E S}=\tau_{i j}^{L E S}-\frac{2}{3} k \delta_{i j}=-2 v_{t} S_{i j} \\
\gamma=-\frac{a_{i j}^{L E S} S_{i j}}{\sqrt{a_{m n}^{L E S} a_{n m}^{L E S} S_{p q} S_{q p}}}, \quad-1<\gamma<1 .
\end{gathered}
$$

A value of -1 implies that the strain rate and anisotropy tensor are aligned in the opposite direction. A value of 0 implies no alignment between the stress and strain rate tensor. Finally, a value of +1 indicates strong alignment between the anisotropy and strain rate tensor. In Fig. 6, it can be seen that a region of poor alignment occurs close to the wall while strong alignment occurs in the wake region behind the slot.

\section{Training Region and Proposed Models}

Using the Machine learning approach discussed earlier, the following models were produced by the algorithm: Models 'KCI.R.1b' 

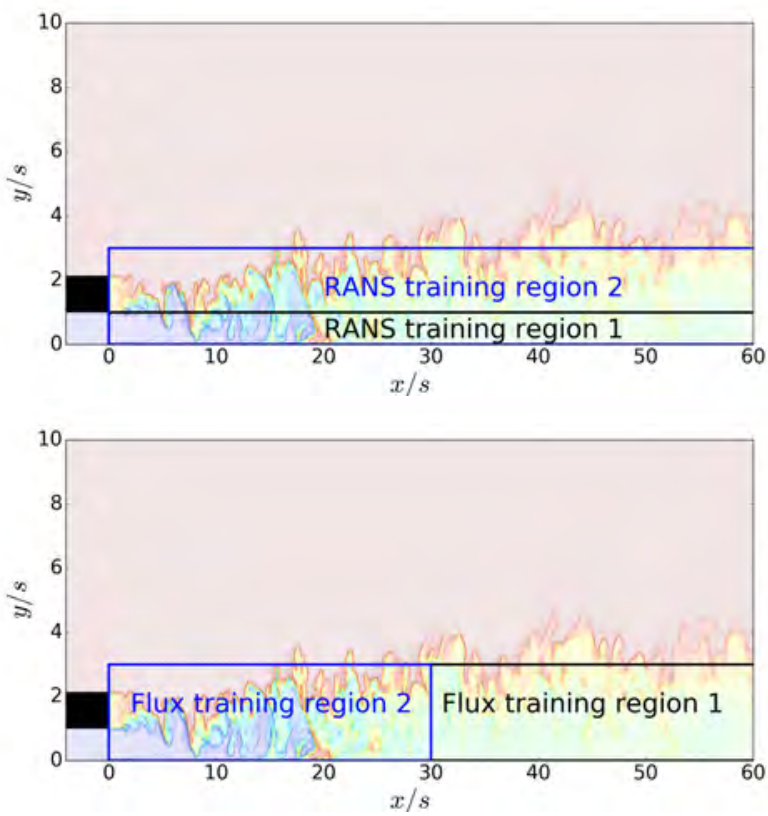

FIGURE 7: Training regions for RANS (top) and heat flux (bottom) models.

and 'KCI.R.thin.1' were trained on the same region illustrated in Fig. 7. The only difference is that model KCI.R.1b is trained on the LES dataset of the thick lip geometry while model KCI.R.thin.1 is trained on the LES dataset of the thin lip geometry. The training region presented here is the region found to give the best improvement in adiabatic wall effectiveness while providing the best match to the LES Reynolds stress profiles. The region was selected based on the high levels of alignment error, shown in Fig. 6. For comparison sake, a separate RANS model that was trained with a less optimal training region is presented (see Tab. 2). For this model (KCI.R.1f), the training region extends up to wall normal height of $3 s$ while the $x$ domain remains the same. A comparison of the alignment between the LES anisotropy and modeled anisotropy, adiabatic wall effectiveness as well as Reynolds stress with the models trained with the optimal region (KCI.R.1b, KCI.R.thin.1) will be presented in the following sections.

$$
\begin{aligned}
a_{i j}^{\text {mod }_{1}=} & V_{i j}^{1}\left(-2 I_{1} I_{2}-1.0 I_{2}-0.43\right) \\
& +V_{i j}^{2}\left(1.0 I_{1} I_{2}+I_{1}+\left(I_{2}\right)^{2}-3.0 I_{2}-3.903\right) \\
& +V_{i j}^{3}\left(4 I_{2}\right) \\
a_{i j}^{\text {mod }_{2}}= & V_{i j}^{1}\left(1.05 I_{1}-0.6\right) \\
& +V_{i j}^{2}\left(2.0 I_{1}-\left(4.0 I_{1}-8.6 I_{2}+8 I_{1} I_{2}-4.3\right)\right. \\
& \left.\quad\left(5.0 I_{1}-4 I_{2}-2 I_{1}^{2}+2 I_{1} I_{2}-2\right)\right) \\
& +V_{i j}^{3}\left(6.0\left(2.0 I_{2}+1.0\right)\left(I_{2}-I_{1}+2.0\right)-1.0\right) \\
a_{i j}^{\text {mod }_{3}}= & V_{i j}^{1}(-0.609)+V_{i j}^{2}\left(-I_{2}-1.0\right)+V_{i j}^{3}\left(-I_{2}\right)
\end{aligned}
$$

In the case of the heat flux models, the training area should be located using the region where either the value of the thermal diffusivity or GGDH coefficient is dominant. In both cases, this region correspond approximately to the domain downstream of the slot lip from $0 \leq y \leq 3$ and $x>0$ (see Fig. 4 and 5). A further consideration is that the selection region should consider only the physical phenomenon for which the mechanism of diffusion occurs primarily due to turbulence and not vortex shedding. In the RANS model training, the magnitude of the anisotropy tensor is not significantly affected by the presence of the additional mechanism of vortex shedding due to the thick lip. The region of large anisotropy occurs mainly at the lower wall region and this is true for both the thick and thin lip case irrespective of the vortex shedding mechanism. On the other hand, the magnitude of the thermal diffusivity or $G G D H$ coefficient is strongly linked to the location of vortex formation. In Fig. 4, it can be seen that the large magnitude of thermal diffusivity occur at the region right behind the thick lip wall. Therefore, the region for which the mechanism of diffusion does not include the vortex shedding effect, $x \approx>30$ and $0 \leq y \leq 3$ (see Fig. 7) is considered for the extraction of the coefficient/thermal diffusivity. For the current flux training region where the models are trained on the LES dataset of the thick lip case, the following models were compared:

$$
\begin{aligned}
\alpha_{t}^{\text {mod }_{1}=} & -2.447 * I_{2}-I T_{1} *\left(-2.21 I_{1}-0.959\right) \\
& +3.447 I T_{1}+3.447 \\
f(. .)^{\bmod _{2}=} & -I_{2} *\left(I_{1}+I T_{2}+0.75\right)-293.25 * I T_{1}+0.75 * I T_{2} \\
& *\left(I T_{7}+16.794\right)+20801.39 * I T_{4}+\left(4 . 9 5 * \left(-4.95 * I_{2}\right.\right. \\
& \left.+4.95) *\left(I_{2}-4.95\right)-41.56\right) *\left(-I_{1}+I T_{1}+2 I T_{3}\right) \\
& +4.4078
\end{aligned}
$$

$$
\begin{aligned}
f(. .)^{\bmod _{3}=} & -2 I_{1}-27.4656 I_{2}-36.2562 I T_{1}-I T_{2} \\
& -I T_{3}+\left(I T_{5}-0.03\right) *\left(-\left(I_{3}-2.488\right) *\left(-I T_{2}\right.\right. \\
& +2.488)+8.129)-\left(7.3603 I T_{8}+97.656\right) *\left(I T_{1}\right. \\
& \left.+5.201 I T_{2}+I T_{3}-0.01\right)+2.003
\end{aligned}
$$

It should be pointed out that for these models, the scalar invariants were extracted from the momentum field computed using the best performing trained RANS model. An overview of the different RANS and flux models is presented in Tab. 2.

\section{Alignment of modeled and LES anisotropy tensors}

In order to test the newly trained models, the alignment of the models with respect to the anisotropy tensor from LES were compared. The alignment of the anisotropy tensors is defined as

$$
\gamma=\frac{a_{i j}^{L E S} a_{i j}^{\text {mod }}}{\sqrt{a_{m n}^{L E S} a_{n m}^{L E S} a_{p q}^{\text {mod }} a_{q p}^{\text {mod }}}}, \quad-1<\gamma<1
$$

In Fig. 8, it can be seen that the alignment results are very similar and in all cases the alignment, particularly close to the lower wall, has significantly improved over the linear models shown in Fig. 6. Models trained on the datasets from the thin or thick lip LES produced a very similar result. This is also the case when the 


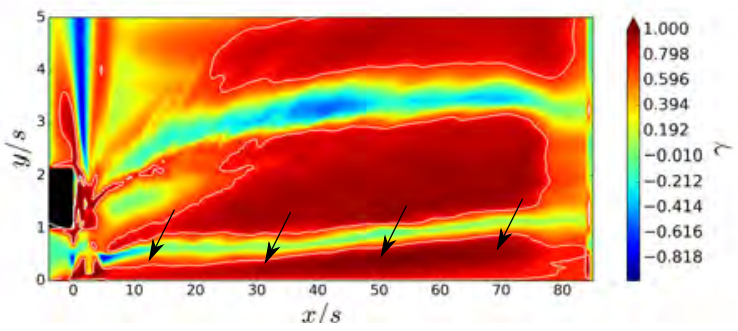

(a) KCI.R.1b model

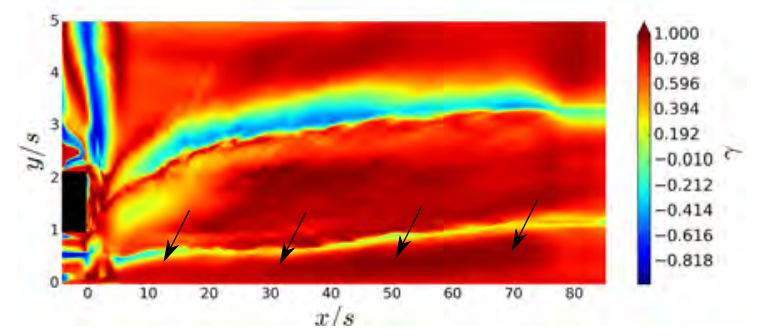

(b) KCI.R.thin. 1 model

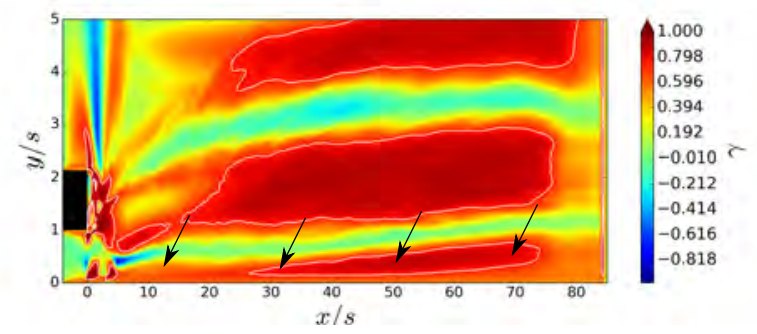

(c) KCI.R.1f model

FIGURE 8: Alignment of strain and anisotropy tensor using different trained non-linear RANS models for $t / s=1.14$ case. Note that arrows are pointing towards the areas of highest interest (close to the lower wall) where significant improvement in the alignment is observed compared to Fig. 6.

models are tested on the thin lip geometry. This implies that the influence of the lip thickness on the anisotropy of the flow field near the lower wall is negligible. In Fig. 8c, the alignment plot of a third model trained under a less optimal region is presented. Although the plot looks very similar to the above two plots, it can be seen that the magnitude of the alignment is comparatively smaller at both the wake and near wall regions.

\section{Reynolds Stress Profiles}

A comparison of the Reynolds Stress profiles at a stream-wise distance of 30 slot heights for the different models are presented in Figs. 9 and 10 for the thick and thin lip cases, respectively. Note that Fig. 10 also shows the results when the models obtained for $B R=1.26$ are used for two lower blowing ratios of the thin lip case. In the comparison of the models, it should be pointed out that the trained models were applied across the entire domain for the active simulation. The analysis of the alignment plot shows that applying the model across the entire domain is appropriate since relatively high alignment is found across the region of interest except for the location near the wall at which a strip of low magnitude of alignment is found (see Fig. 8). This location correspond closely to the wall normal height for which the growing boundary layer interacts with the wake behind the lipwall. At this specific location, it was found that neither of the trained models were able to adequately recover a high alignment value at the intersection. (a)
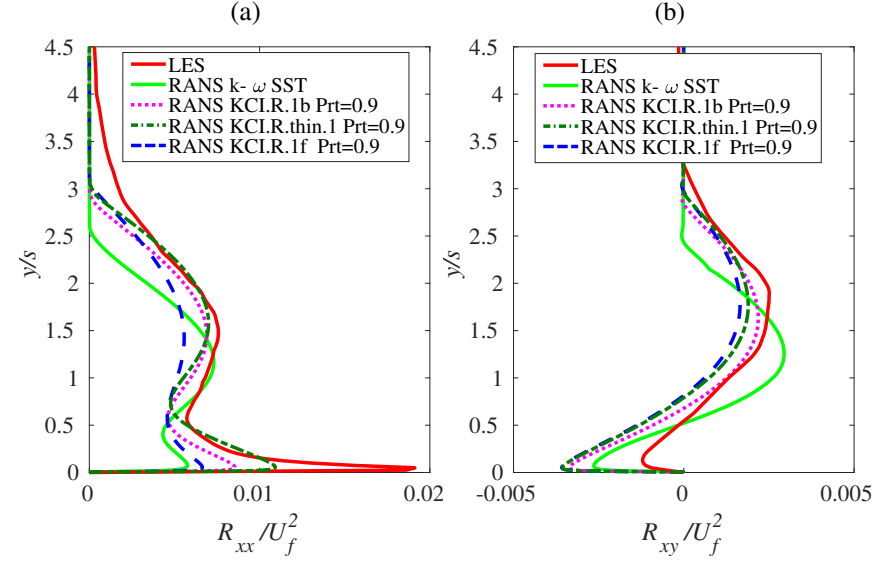

FIGURE 9: $R_{x x}$ (a) and $R_{x y}$ (b) from trained models compared with baseline RANS and LES profiles at $x / s=30$ for $t / s=1.14$.

In Figs. 9 and 10, it can be seen that there is significant improvement in the prediction of the $R_{x x}$ component when compared to the baseline RANS model. In the near wall region, the trained RANS models are able to produce a sharper peak as compared to the baseline RANS model. It should also be noted in Fig. 10b-c that the prediction of the $R_{x x}$ component for other blowing ratios of the thin lip case is significantly improved compared to the baseline RANS model even using the current models trained at $B R=1.26$. This implies the applicability of the models for different conditions than what they were trained on. The $R_{x y}$ component is better predicted for model KCI.R.1b and KCI.R.thin. 1 in the case of the thicker slot, although both models performed poorer in the case of the thin lip as compared to the baseline RANS model in terms of overall magnitude. However, the general shape of the profile is still captured with the trained models. Results from the model trained in a different region, KCI.R.1f, are included in Fig. 9 as well and show that for both components of the Reynolds stress tensor, particularly $R_{x x}$, the improvement over the baseline model is not as good as for the other models, suggesting that training region 1 (see Fig. 7, top) produces better results.

\section{Comparison of modelled and LES Heat Flux Vector}

The trained heat flux models are now tested a-priori using the constructed invariants from the converged RANS field to compare the modeled heat flux to the actual heat flux vector from the LES dataset. In a flow domain for which the temperature gradient is dominant in the $y$ direction, the streamwise heat flux tends to be under predicted using the eddy-diffusivity model. Using a model based on the generalized gradient diffusion hypothesis would in theory give better prediction of the heat flux especially in the streamwise direction. In this comparison, the modeled form of the heat flux vector can take either the form of the gradient diffusion hypothesis or the generalized gradient diffusion hypothesis seen in equations (11) and (12), respectively.

From Fig. 11a and 12a, it can be inferred that the mechanism driving the decrease of the wall temperature is due to both the negative streamwise and wall normal heat flux. It is also evident that the wall-normal heat flux is in general larger than the streamwise heat flux, especially in the wake region behind the lip wall. The dominant region where the flux is largely negative is mostly concentrated 
(a) $\mathrm{BR}=1.26$
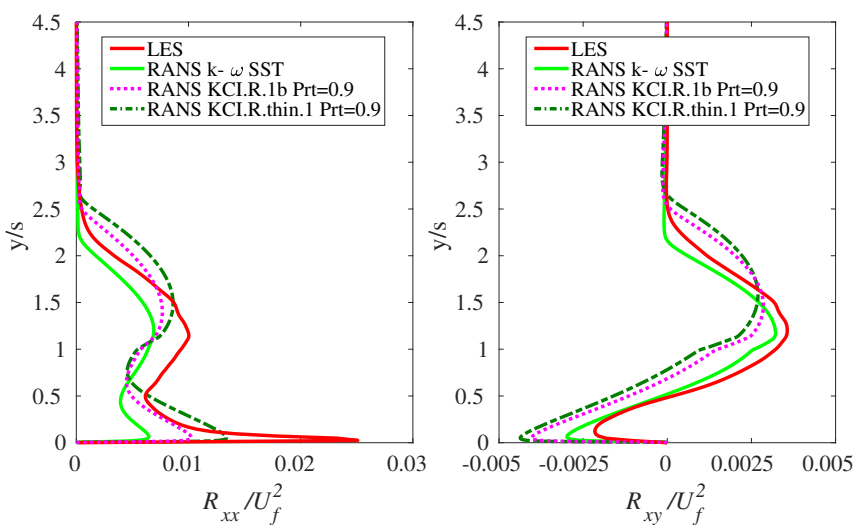

(b) $\mathrm{BR}=1.07$
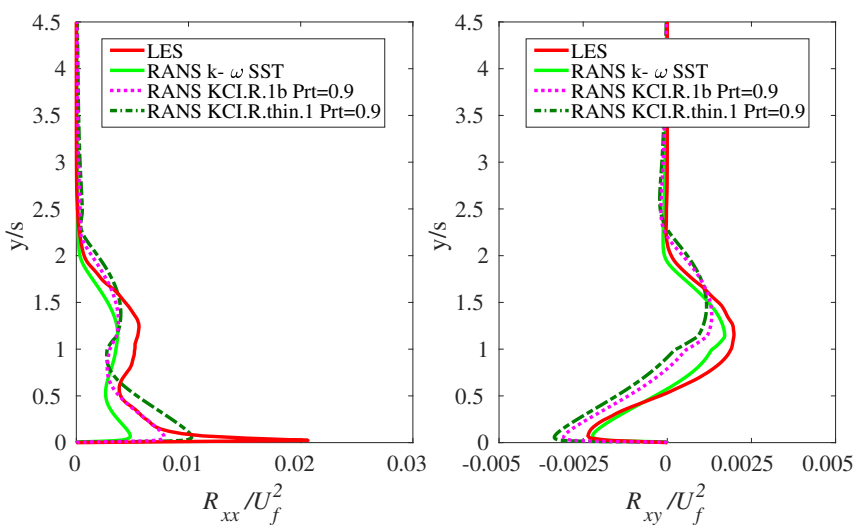

(c) $\mathrm{BR}=0.86$
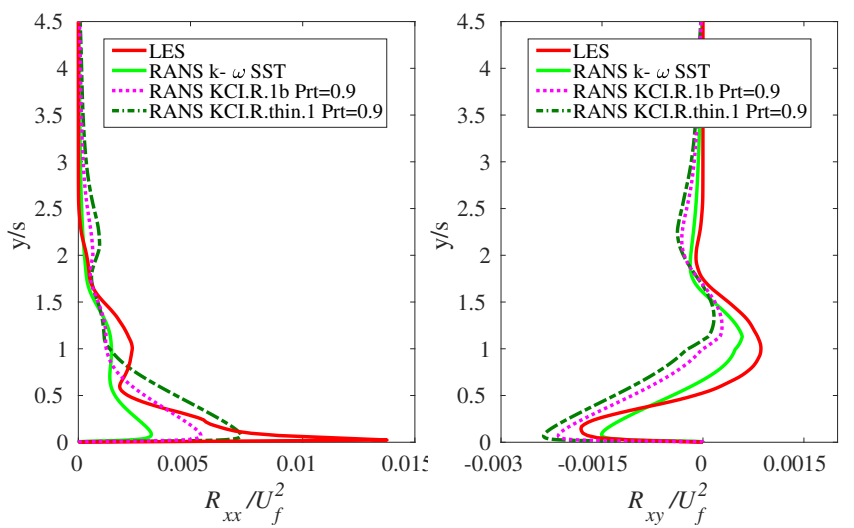

FIGURE 10: $R_{x x}$ (left) and $R_{x y}$ (right) from trained models compared with baseline RANS and LES profiles at $x / s=30$ for $t / s=$ 0.126 at different blowing ratios: (a) $\mathrm{BR}=1.26$, (b) $\mathrm{BR}=1.07$ and (c) $\mathrm{BR}=0.86$.

in a region right behind the lipwall and away from the lower wall. In the vicinity of the lower wall, both the streamwise and wall-normal heat flux components are much smaller in magnitude although the wall-normal heat flux play a greater role in the transport of heat away from the wall. Heat flux models based on the gradient diffusion hypothesis (HF.1) appear to be unable to produce the correct magnitude of the streamwise heat flux component without over predicting wall-normal heat flux. With respect to the LES heat flux, it can be seen that the heat flux produced by HF.1 model over predicts the wall-normal and under predicts the streamwise heat flux. In contrast, the machine learnt heat flux model (HF.2) based on the generalized gradient diffusion hypothesis is able to provide the best

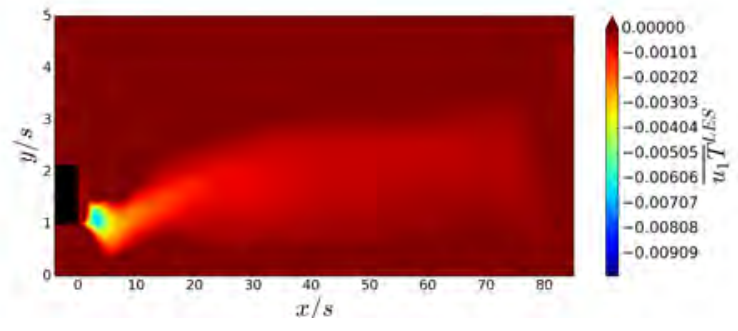

(a) LES streamwise heat flux

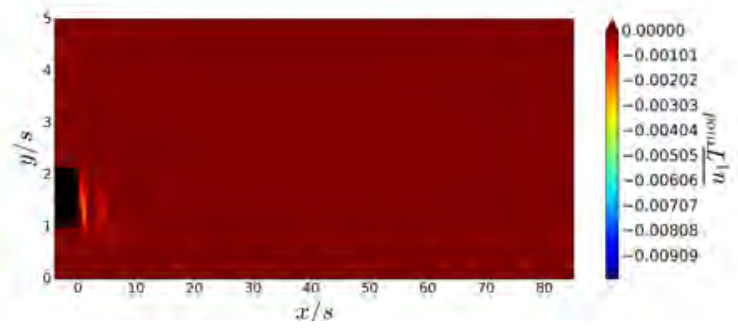

(b) HF.1 streamwise heat flux

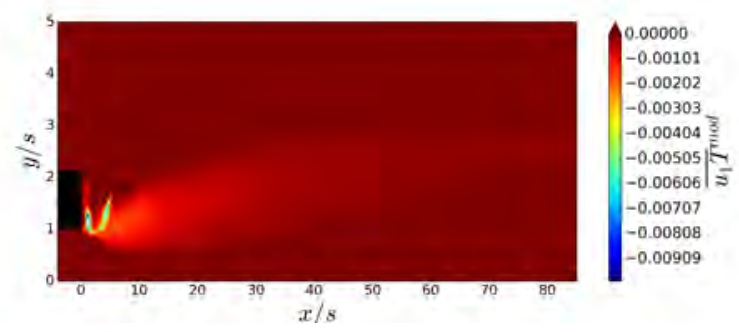

(c) HF.2 GGDH streamwise heat flux

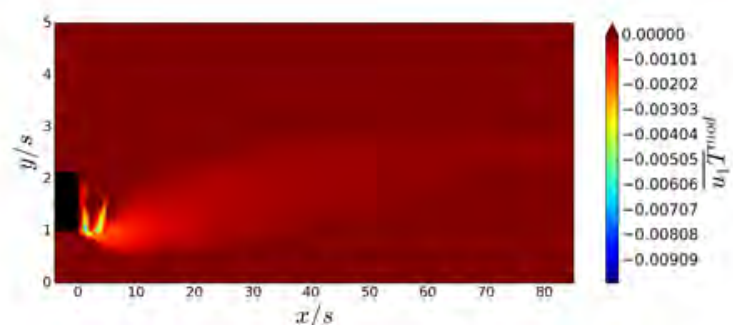

(d) HF.3 GGDH streamwise heat flux

FIGURE 11: Comparison of streamwise heat flux obtained from different flux models for case $t / s=1.14$.

prediction of both the streamwise and wall-normal heat flux components when compared to the LES data, especially the near-lip wall region. To illustrate the effect of a change in training region, another flux model was trained using a domain between $0<x / s<30$ instead. A comparison of the adiabatic wall effectiveness obtained from the above-mentioned heat flux models is made in the next section.

\section{Adiabatic Wall Effectiveness of Trained Models}

In Fig. 13a it can be seen that the adiabatic wall effectiveness of the cases using the KCI.R.1b and KCI.R.thin.1 RANS models show significant improvement over the baseline $k-\omega$ SST model, resulting in a very good comparison with both LES and the experiment [1]. Of particular interest is that the model KCI.R.1b, trained using the thick-lip LES dataset, produces almost the same adiabatic wall effectiveness as the model KCI.R.thin.1, trained using the thin-lip LES dataset. Further, applying the models trained using 


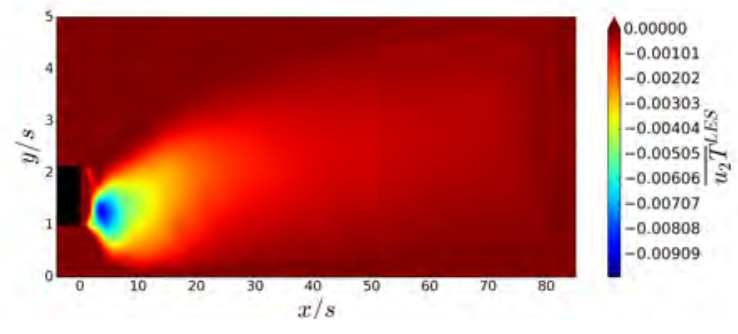

(a) LES wall-normal heat flux

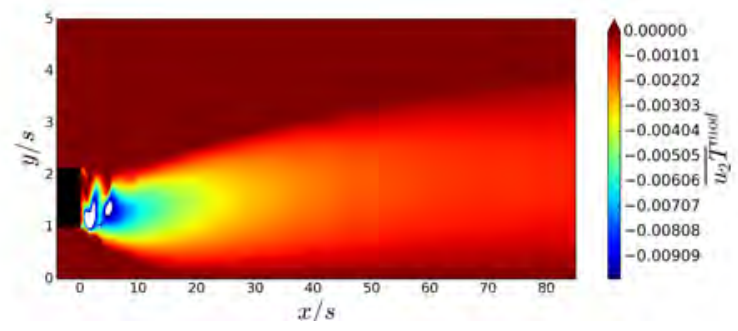

(b) HF.1 wall-normal heat flux

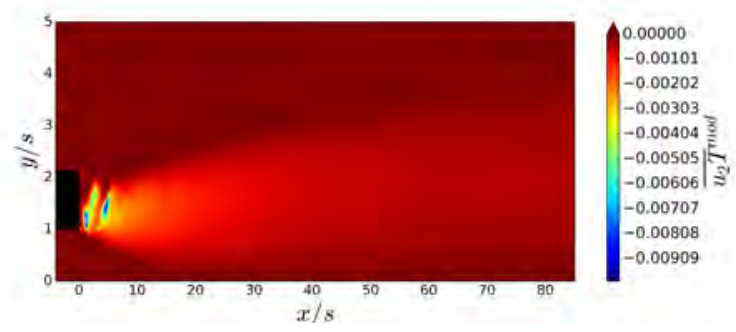

(c) HF.2 GGDH wall-normal heat flux

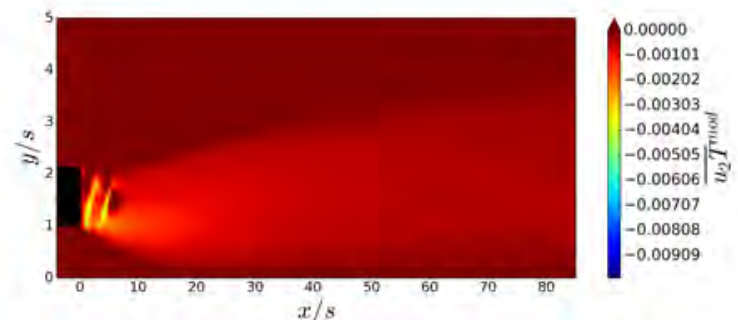

(d) HF.3 GGDH wall-normal heat flux

FIGURE 12: Comparison of wall-normal heat flux obtained from different flux models for case $t / s=1.14$.

the LES dataset at $B R=1.26$ to different blowing ratios of the thin lip geometry leads to a significant improvement in RANS prediction of adiabatic wall effectiveness, as shown in Fig. 13b-c. Taken together, these results suggest that the developed models, using the same training region and blowing ratio, are robust to changes in geometry and flow condition.

However, when performing RANS using the newly trained models on the thick-lip case with $t / s=1.14$, a much wider spread of the results can be observed (see Fig. 14). Using models KCI.R.1b, KCI.R.thin.1 and KCI.R.1f again an improvement over the baseline model results can be observed, with little difference between the models. However, the results are by far not as close to the reference data as for the thin-lip case. This is mainly due to the fact that in the thick-lip geometry the error in the scalar flux model is more significant than in the thin-lip case. Thus for the thick-lip case training only a RANS model is not sufficient and additionally a trained heat-flux model needs to be considered. Figure 14 also includes the results from the combined RANS and flux (a) $\mathrm{BR}=1.26$

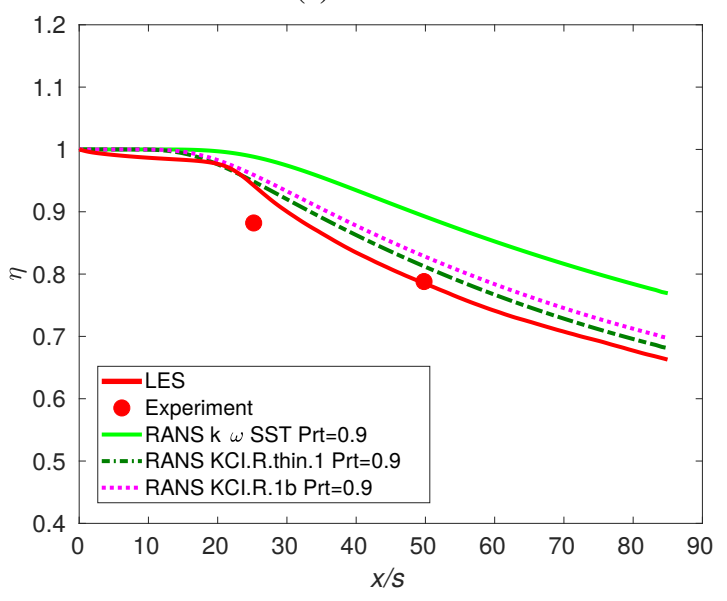

(b) $\mathrm{BR}=1.07$

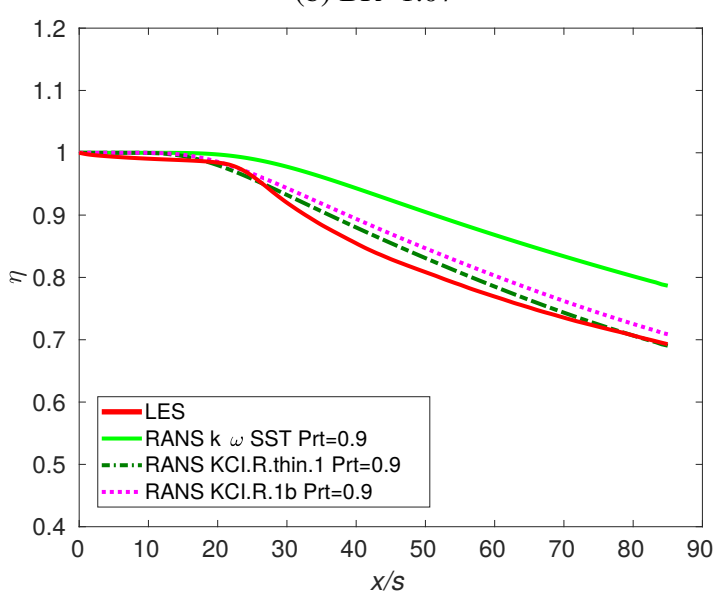

(c) $\mathrm{BR}=0.86$

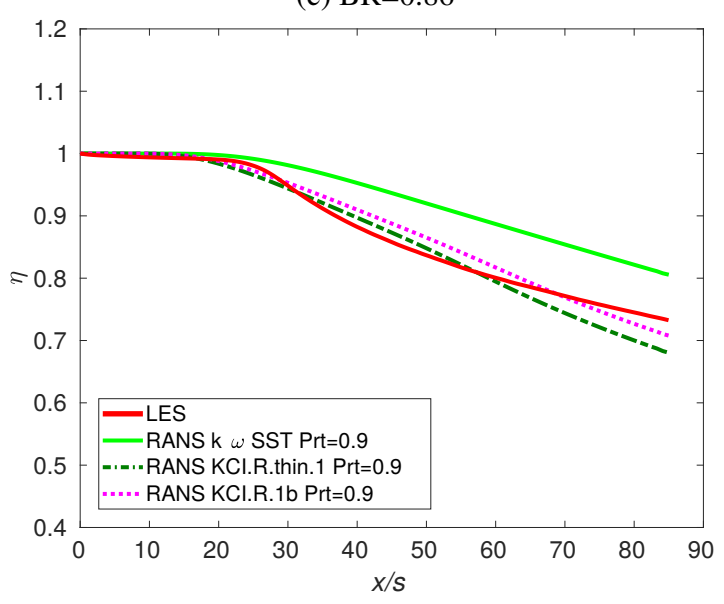

FIGURE 13: Comparison of $\eta_{\text {wall }}$ for different RANS models for $t / s=0.126$ at different blowing ratios: (a) $\mathrm{BR}=1.26$, (b) $\mathrm{BR}=1.07$ and (c) $\mathrm{BR}=0.86$.

models KCI.R.1b-HF.1, KCI.R.1b-HF.2, KCI.R.1b-HF.3. For all three trained heat-flux models, combined with the most successful RANS model KCI.R.1b, the results improve further over the baseline model and the trained RANS-only model cases. A comparison of the $G D H$ model, KCI.R.1b-HF.1, and the GGDH model, KCI.R.1b-HF.2, shows that the correct prediction of the streamwise heat flux just in the wall region downstream of the lip is necessary to obtain a better match in adiabatic wall effectiveness to the LES results. In particular KCI.R.1b-HF.2 succeeds in approximating the 


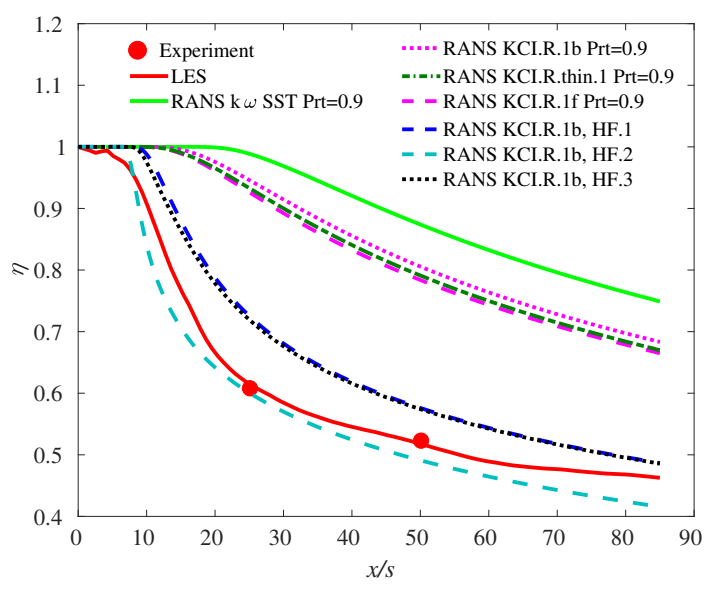

FIGURE 14: Comparison of $\eta_{\text {wall }}$ for different RANS and heat flux models for $t / s=1.14$.

reference data very closely. Different $G D H$ models were generated using different training regions and it was found that none of these models were able to match the LES results closely, although they still performed significantly better than results based on the standard eddy-diffusivity model. The large difference between models KCI.R.1b-HF.2 and KCI.R.1b-HF.3, trained in different regions, shows that the adiabatic wall effectiveness is very sensitive to the change in the flux model training region as the temperature field is explicitly dependent on the modelled heat flux term in its transport equation.

Overall, the models presented so far therefore are able to significantly improve the prediction of the adiabatic wall effectiveness for both geometries, although with one caveat. When using the most successful Reynolds stress model developed for the thick-lip case (equation 16a) combined with the heat flux model (equation $17 b$ ), denoted KCI.R.1b-HF.2, on the thick-lip case, the adiabatic wall effectiveness is predicted well (see Fig. 14). However, on the other hand, when using the best model combination obtained for the thick-lip case, KCI.R.1b-HF.2, on the thin-lip case, $\eta_{\text {wall }}$ is under predicted (not shown in Fig. 13). This behavior can be explained by having another look at the instantaneous temperature fields shown in Fig. 2. For the thick trailing-edge case, low-frequency vortex shedding is produced by the trailing edge that persists for a large distance downstream, while in the thin trailing-edge case the vortex shedding frequency is higher and does not persist as long downstream, in particular when looking at the URANS result. For the thin-lip case, it was also found that the error in heat flux modelling between baseline RANS and LES was smaller than in the thick lip case. This implies that by training a RANS model that can produce a flow field that more closely matches the reference data might suffice in order to obtain a good prediction of $\eta_{\text {wall }}$, as indeed observed in Fig. 13. On the other hand, for the thick-lip case, a heat flux model needs to be trained in conjunction with a RANS model to improve $\eta_{\text {wall }}$ predictions. However, as trained RANS models are not able to predict an accurate flow field, as indicated by the Reynolds stress components, see Figs. 9- 10, the trained heat flux models are at risk of overcompensating in order to obtain the correct temperature field based on an inaccurate flow field. In order to address this issue, a future study using unsteady RANS will be conducted with the aim of obtaining accurate flow and temperature fields for different geometries using only one set of trained models.

\section{Conclusion}

A Gene Expression Programming based machine-learning technique has been applied for the development of non-linear turbulence and heat transfer closure models suited for steady RANS calculations of a fundamental trailing-edge slot. Highly resolved large-eddy simulations were initially performed for two different trailing-edge slot thicknesses and were shown to agree well with experimental data [1], thus providing suitable training data for model development. RANS calculations using a linear stress-strain model and eddy-diffusivity based heat flux models were conducted of the same cases for comparison, showing the well known over prediction of adiabatic wall effectiveness. The LES data were used to identify deficiencies in the baseline RANS calculations. It was shown that the turbulent stress tensor is poorly aligned with the strain rate tensor, in particular in the near wall region, justifying the use of the mean squared error of the anisotropy tensor as a cost function for the RANS model development, and explaining the use of the nearwall region as most successful training region.

The machine-learnt models were then applied to both thin and thick trailing-edge slot geometries to assess their performance. The comparison of the Reynolds stress profiles show that the trained RANS models are able to provide somewhat better prediction of the normal components of the Reynolds stress tensor in both the thick and thin lip cases when compared with the baseline results, without penalty of performance for the shear component. However, importantly, it is found that the trained turbulence model in conjunction with the standard eddy-diffusivity model can achieve a good match with the reference data for the thin lip geometry in terms of the adiabatic wall effectiveness. This was also the case when this combination (i.e. the RANS model trained using the LES dataset at $B R=1.26$ and the standard eddy-diffusivity model) was applied to different blowing ratios of the thin lip geometry. The trained non linear models also improve the prediction of adiabatic wall effectiveness for the thick-lip case, although the results do not agree well with the reference data.

Extracting the thermal diffusivity from the LES data revealed significant spatial gradients that cannot be represented by an eddydiffusivity model, thus motivating the use of the GEP algorithm for turbulent heat flux model development. For the turbulent heat flux models, the strain rate and vorticity tensors and the temperature gradient vector were used to construct the basis functions. The LES data showed that the streamwise heat flux is the least important component. It was found that a modeled form of the heat flux vector based on the generalized gradient diffusion hypothesis provided a better match to the LES heat flux especially in terms of the streamwise heat flux component. This resulted in good predictions of the adiabatic wall effectiveness even for the thick lip case when using the trained heat flux model with a non-linear trained RANS closure. Thus, a set of models has been developed and presented, each producing accurate predictions of the adiabatic wall effectiveness for the respective, topologically different, cases of thick and thin trailing edges.

Applying the combination of non-linear turbulence and heat flux models best suited for the thick lip case to the thin lip case, an under prediction of the adiabatic wall effectiveness is found. This is most likely due to the fact that the steady RANS does not feature the large-scale vortex shedding present in the thick trailing edge case and thus the heat flux model developed for the thick-lip case is overcompensating for the lack of mixing due to the deterministic unsteadiness. To address this, the next logical step in developing a machine-learning framework for non-linear turbulence and heat 
flux modeling is the consideration of unsteady RANS and models developed specifically in that context.

\section{ACKNOWLEDGMENT}

This work was supported by resources provided by the Pawsey Supercomputing Centre with funding from the Australian Government and the Government of Western Australia. The University of Melbourne authors also acknowledge the financial support and the permission to publish by General Electric.

\section{NOMENCLATURE}

$a_{i j}$ non-dimensional anisotropy tensor

$c_{\mu} \quad$ turbulence model constant; 0.09

$I_{\text {slot }}$ inlet slot turbulence intensity

$I_{\text {main }}$ inlet main turbulence intensity

$k$ turbulent kinetic energy

$l_{t}$ turbulent length scale

$\operatorname{Pr}$ Prandtl number

$\mathrm{Pr}_{t} \quad$ turbulent Prandtl number

$S_{i j} \quad$ strain rate tensor

$U_{b}$ inlet slot bulk velocity

$U_{f s}$ inlet main free-stream velocity

$\alpha_{t} \quad$ Thermal Diffusivity

$\eta_{\text {wall }}$ adiabatic wall effectiveness, $\frac{T_{\text {wall }}-T_{f s}}{T_{\text {slot }}-T_{f s}}$

$v_{t}$ turbulent viscosity

$v$ kinematic viscosity

$\rho$ density

$\tau_{i j} \quad$ Reynolds stress tensor

$\Omega_{i j} \quad$ rotation rate tensor

\section{Abbreviations}

$B R$ blowing ratio

$G D H$ gradient diffusion hypothesis

$G G D H$ generalised gradient diffusion hypothesis

\section{Superscripts and Subscripts}

$f s$ free-stream

LES LES quantity

mod modeled quantity

$s$ slot height

$t$ slot lip thickness

$w$ wall

\section{REFERENCES}

[1] Whitelaw, S., and Kacker, J., 1963. “An Experimental Investigation of the influence of slot lip thickness of the impervious wall effectiveness of the uniform density, two dimensional wall jet". International Journal of Heat and Mass Transfer, 542, pp. 1196-1201.

[2] Medic, G., and Durbin, P. A., 2005. "Unsteady Effects on Trailing Edge Cooling”. Journal of Heat Transfer, 127(4), pp. 388-392.

[3] Joo, J., and Durbin, P. A., 2009. "Simulation of turbine blade trailing edge cooling". Journal of Fluids Engineering, Transactions of the ASME, 131(2), pp. 211021-2110214.
[4] Ling, J., Kurzawski, A., and Templeton, J., 2016. "Reynolds averaged turbulence modelling using deep neural networks with embedded invariance". Journal of Fluid Mechanics, 807, pp. 155-166.

[5] Parish, E. J., and Duraisamy, K., 2016. "A paradigm for datadriven predictive modeling using field inversion and machine learning". Journal of Computational Physics, 305, pp. 758774.

[6] Ferreira, C., 2001. "Gene expression programming: A new adaptive algorithm for solving problems". Complex Systems, 13(2), pp. 87-129.

[7] Weatheritt, J., and Sandberg, R. D., 2016. "A novel evolutionary algorithm applied to algebraic modifications of the RANS stress-strain relationship". Journal of Computational Physics, 325, pp. 22-37.

[8] Weatheritt, J., Sandberg, R. D., Ling, J., Saez, G., and Bodart, J., 2017. "A comparative study of contrasting machine learning frameworks applied to RANS modeling of jets in crossflow”. In ASME Turbo Expo 2017: Turbomachinery Technical Conference and Exposition, American Society of Mechanical Engineers, p. V02BT41A012.

[9] Weatheritt, J., Pichler, R., Sandberg, R. D., Laskowski, G. M., and Michelassi, V., 2017. "Machine learning for turbulence model development using a high fidelity HPT cascade simulation". In ASME Turbo Expo 2017: Turbomachinery Technical Conference and Exposition, American Society of Mechanical Engineers, p. V02BT41A015.

[10] Duraisamy, K., and Durbin, P., 2014. "Transition modeling using data driven approaches". In Proceedings of the CTR Summer Program, p. 427.

[11] Zhang, Z. J., and Duraisamy, K., 2015. "Machine learning methods for data-driven turbulence modeling". In 22nd AIAA Computational Fluid Dynamics Conference, p. 2460.

[12] Milani, P. M., Ling, J., Saez-Mischlich, G., Bodart, J., and Eaton, J. K., 2018. "A machine learning approach for determining the turbulent diffusivity in film cooling flows". Journal of Turbomachinery, 140(2), p. 021006.

[13] Sandberg, R., 2015. "Compressible-flow DNS with application to airfoil noise". Flow, Turbulence and Combustion, 95(2-3), pp. 211-229.

[14] Ducros, F., Nicoud, F., and Poinsot, T., 1998. "Wall-adapting local eddy-viscosity models for simulations in complex geometries". Conference on Numerical Methods in Fluid Dynamics, pp. 1-7.

[15] Ivanova, E., and Laskowski, G. M., 2014. "LES and Hybrid RANS/LES of a fundamental trailing edge slot". In ASME Turbo Expo 2014: Turbine Technical Conference and Exposition, American Society of Mechanical Engineers, p. V05BT13A030.

[16] Sandberg, R. D., and Sandham, N. D., 2006. "Nonreflecting zonal characteristic boundary condition for direct numerical simulation of aerodynamic sound". AIAA journal, 44(2), pp. 402-405.

[17] Menter, F., and Esch, T., 2001. "Elements of industrial heat transfer predictions". In 16th Brazilian Congress of Mechanical Engineering (COBEM), Vol. 109.

[18] Leschziner, M., 2015. Statistical turbulence modelling for fluid dynamics-demystified: an introductory text for graduate engineering students. World Scientific.

[19] Pope, S. B., 1975. "A more general effective-viscosity hypothesis". Journal of Fluid Mechanics, 72(2), pp. 331-340. 
[20] Spalart, P., Shur, M., Strelets, M. K., and Travin, A., 2015. "Direct simulation and RANS modelling of a vortex generator flow". Flow, Turbulence and Combustion, 95(2-3), pp. 335350 .

[21] Koza, J. R., 1992. Genetic programming: on the programming of computers by means of natural selection, 1 ed. MIT press.

[22] Daly, B., and Harlow, F., 1970. "The generalized gradient diffusion hypothesis". Phys. Fluids, 13, p. 2634.

[23] Schmitt, F. G., 2007. “About Boussinesq's turbulent viscosity hypothesis: historical remarks and a direct evaluation of its validity". Comptes Rendus - Mecanique, 335(9-10), pp. 617627. 


\section{University Library}

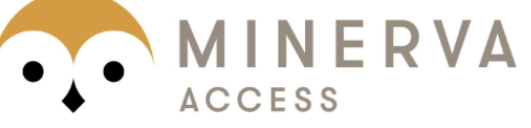

A gateway to Melbourne's research publications

Minerva Access is the Institutional Repository of The University of Melbourne

Author/s:

Sandberg, RD;Tan, R;Weatheritt, J;Ooi, A;Haghiri, A;Michelassi, V;Laskowski, G

Title:

Applying Machine Learnt Explicit Algebraic Stress and Scalar Flux Models to a Fundamental Trailing Edge Slot

Date:

2018

\section{Citation:}

Sandberg, R. D., Tan, R., Weatheritt, J., Ooi, A., Haghiri, A., Michelassi, V. \& Laskowski, G. (2018). Applying Machine Learnt Explicit Algebraic Stress and Scalar Flux Models to a Fundamental Trailing Edge Slot. Proceedings of the ASME Turbo Expo 2018:

Turbomachinery Technical Conference and Exposition (GT2018), 5A-2018, American Society of Mechanical Engineers. https://doi.org/10.1115/GT2018-75444.

Persistent Link:

http://hdl.handle.net/11343/241853 